Annuaire suisse de politique de développement

4 | 1984

Annuaire Suisse - Tiers Monde 1984

\title{
III. Coopération au développement
}

Philippe Besson

\section{OpenEdition}

Journals

Édition électronique

URL : http://journals.openedition.org/aspd/1179

DOI : $10.4000 /$ aspd. 1179

ISSN : 1663-9669

\section{Éditeur}

Institut de hautes études internationales et du développement

\section{Édition imprimée}

Date de publication : 1 janvier 1984

Pagination : 89-111

ISSN : 1660-5934

\section{Référence électronique}

Philippe Besson, «III. Coopération au développement », Annuaire suisse de politique de développement

[En ligne], 4 | 1984, mis en ligne le 03 février 2013, consulté le 08 septembre 2020. URL : http:// journals.openedition.org/aspd/1179; DOI : https://doi.org/10.4000/aspd.1179 


\section{COOPERATION AU DEVELOPPEMENT}

En 1983-1984, l'aide publique au développement est restée un domaine largement débattu de la politique fédérale. A la fin de 1983, la pétition "Aider aujourd'hui pour survivre demain", lancée par sept organismes d'entraide et qui a recueilli plus de 200.000 signatures, a été remise aux Chambres fédérales. La pétition avait pour but de faire revenir les Chambres sur l'inclusion de l'APD dans les mesures d'économie de la Confédération. Le Conseil des Etats a déjà examiné la pétition au début de 1984 et l'a transmise au Conseil fédéral avec la recommandation d'en tenir compte, dans la mesure du possible, dans ses futurs plans. Le Conseil national a abordé cet objet dans sa session d'automne 1984.

Enfin, le Conseil fédéral a présenté, le 19 mars 1984, son Message sur la continuation de la coopération technique et de l'aide financière en faveur des pays en développement, qui propose aux Chambres l'adoption d'un créditcadre de 1,8 milliard de francs réparti sur une période minimum de trois ans. Cet objet a été discuté par le Conseil des Etats en été 1984 et par le Conseil national en automne.

\section{LES DEPENSES DE LA COOPERATION AU DEVELOPPEMENT EN 1983 (AIDE PUBLIQUE AU DEVELOPPEMENT)}

En 1983, la Suisse (Confédération, cantons et communes) a consacré 574,3 millions de francs à la coopération au développement, ce qui représente $0,27 \%$ du PNB (1982 : 0,24\%). Toutefois, le recours au paiement différé de certaines contributions (paiement par "notes") et la méthode statistique actuellement en vigueur à I'OCDE pour refléter cette procédure permettront de faire figurer le chiffre de $0,31 \%$ du PNB comme indicateur de I'APD de la Suisse pour 1983. (Cf. partie Statistiques, chap. 3 "Aide publique au développement".)

Les dépenses de la seule Confédération se sont élevées en 1983 à 565,9 millions de francs, soit environ à 87 francs par habitant. La Confédération annonce depuis 1982, dans sa communication à I'OCDE, le montant des dépenses encourues au titre de l'administration des programmes, lequel est incorporé au montant de I'APD de tous les pays membres du CAD de I'OCDE. En 1983, ce montant s'est élevé à environ 15 millions de francs. Pour 1984, le budget fédéral a prévu des dépenses de l'ordre de 598 millions de francs y compris 17 millions consacrés aux mesures au titre du programme de relance. 
Le tableau ci-dessous présente la planification financière pour la législature en cours :

\section{PLANIFICATION FINANCIERE 1984-87 \\ DE L'AIDE AU DEVELOPPEMENT DE LA CONFEDERATION}

(Dépenses en millions de francs et en pourcentage du PNB)

\begin{tabular}{|c|c|c|c|c|c|}
\hline & 1984 & 1985 & 1986 & 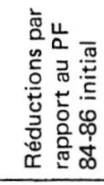 & 1987 \\
\hline $\begin{array}{l}\text { 1. Plan financier } 1984-85 \text { du } 5.10 .81 \\
\text { et demandes des Départ. pour } \\
1986 \text { en mai } 1982\end{array}$ & $\begin{array}{c}710 \\
(0,31 \%)\end{array}$ & $\begin{array}{c}800 \\
(0,34 \%)\end{array}$ & $\begin{array}{c}968 \\
(0,39 \%)\end{array}$ & & \\
\hline $\begin{array}{l}\text { 2. Plan financier } 1984-85 \text { après } \\
\text { coupures linéaires } 10 \%\end{array}$ & 639 & 718 & 879 & & \\
\hline $\begin{array}{l}\text { Mesures d'assainissement de } \\
380 \text { mio fr. proposés dans le } \\
\text { Rapport sur la planification } \\
\text { financière } 1984-85 \text { du } 4.10 .82\end{array}$ & -50 & -100 & -230 & -300 & \\
\hline 3. Plan financier du 4.10 .82 & $\begin{array}{c}589 \\
(0,26 \%)\end{array}$ & $\begin{array}{c}618 \\
(0,26 \%)\end{array}$ & $\begin{array}{c}649 \\
(0,26 \%)\end{array}$ & & \\
\hline $\begin{array}{l}\text { 4. Décision du Conseil fédéral du } \\
16.11 .83 \text { (après coupures } \\
\text { linéaires } 10 \% \text { ) }\end{array}$ & $\begin{array}{c}581 \\
(0,26 \%)\end{array}$ & $\begin{array}{c}632 \\
(0,27 \%)\end{array}$ & $\begin{array}{c}689 \\
(0,28 \%)\end{array}$ & & $\begin{array}{c}782 \\
(0,31 \%)\end{array}$ \\
\hline $\begin{array}{l}\text { Mesures au titre du } \\
\text { programme de relance }\end{array}$ & +17 & +16 & +26 & & +13 \\
\hline $\begin{array}{l}\text { 5. Plan financier } 1985-86 \text { du } 18.1 .84 \\
\text { (après coupures linéaires } 10 \% \\
\text { et y compris mesures ad titre } \\
\text { du programme de relance) }\end{array}$ & $\begin{array}{c}598 \\
(0,27 \%)\end{array}$ & $\begin{array}{c}648 \\
(0,28 \%)\end{array}$ & $\begin{array}{c}715 \\
(0,30 \%)\end{array}$ & -275 & $\begin{array}{c}795 \\
(0,31 \%)\end{array}$ \\
\hline
\end{tabular}

\section{Notes}

Les chiffres ci-dessus se rapportent à I'APD relevant de crédits de programme, non compris les frais administratifs ( $c$ 'est-à-dire légèrement inférieurs aux montants de la rubrique 152 de la classification fonctionnelle).

Les réductions par rapport au PF 84-86 initial représentent les coupures supplémentaires opérées en plus des coupures linéaires de $10 \%$. 
Les quatre principaux crédits de programme actuellement en cours sont les suivants :

- le crédit de programme concernant la continuation de la coopération technique et de l'aide financière de 1.650 millions de francs du 8 décembre 1980 , entré en vigueur le 1er avril 1981;

- le crédit de programme de 300 millions de francs relatif à la participation de la Suisse à l'augmentation du capital des Banques asiatique, interaméricaine et africaine de développement du 26 septembre 1979, entré en vigueur le 1er octobre 1979 pour une période minimale de quatre ans;

- le crédit de programme de 350 millions de francs destiné au financement de mesures de politique économique et commerciale au titre de la coopération internationale au développement, ouvert le 14 décembre 1981 pour la période allant du 1er juillet 1982 au 30 juin 1985 au moins. Une augmentation de 100 millions de francs a été approuvée le 14 mars 1983 pour assurer le financement complémentaire de crédits mixtes et d'aides à la balance des paiements dans le cadre du programme de relance visant au renforcement de l'économie suisse;

- le crédit de programme de 360 millions de francs concernant la continuation de l'aide humanitaire internationale, approuvé le 3 décembre 1981, pour une période de trois ans au minimum à compter du 1er avril 1982.

D'autres contributions sont effectuées au titre de l'aide publique au développement sur la base de crédits d'engagement annuels :

- la contribution ordinaire de la Confédération au Comité international de la Croix-Rouge (CICR) et la participation de la Suisse au budget administratif du Comité intergouvernemental pour les migrations (CIM), fondées respectivement sur l'arrêté fédéral (AF) du 1er décembre 1981 et I'AF du 17 mars 1954;

- les bourses octroyées à des étudiants de pays en développement se formant dans des universités suisses, selon la modification du 7 octobre 1983 de I'AF du 19 décembre 1980.

\section{Répartition des crédits}

En 1983, la part des dépenses fédérales consacrée à la coopération technique a été de $45,9 \%(1982: 46,8 \%)$. La part des dépenses consacrée à l'aide humanitaire et alimentaire a été de $21,8 \%(1982: 18,7 \%)$. Les dépenses au titre des mesures économiques et commerciales ont représenté en 1983 $12,1 \%$ du total des dépenses fédérales d'APD (1982 : 6,6\%).

La part des dons a été en 1983, par rapport aux prêts, de $92 \%$ (1982 : $94 \%)$. Ce léger fléchissement s'explique en partie par l'accroissement des crédits mixtes.

On constate que les pays les moins avancés (PMA) ont reçu $34 \%$ de I'APD bilatérale de la Suisse (1982: $36 \%$ ). Si I'on considère ensemble le groupe des PMA et celui des autres pays à faible revenu (PNB/habitant inférieur à $600 \$$ ), on constate que $66 \%$ des dépenses leur ont été consacrés (1982: $67 \%$ ). 
La part de l'aide bilatérale aux dépenses d'APD a été en 1983 de $74 \%$; celle de l'aide multilatérale a été de $26 \%$.

L'Afrique reste en tout cas le continent au centre des préoccupations de la Suisse. La sécheresse dans les pays du Sahel reste extrêmement préoccupante, de sorte que la Suisse consacre une partie importante de ses moyens en collaborant avec le Comité permanent inter-Etats de lutte contre la sécheresse au Sahel (CILSS).

En conclusion, la répartition de l'aide évolue en gros assez peu en dehors de la croissance des fonds attribués aux mesures économiques et financières, qui pourrait cependant n'être que conjoncturelle.

\section{LES POINTS SAILLANTS DES DEBATS PARLEMENTAIRES SUR LA COOPERATION AU DEVELOPPEMENT}

A l'occasion des débats aux Chambres sur le budget et le programme d'économies, deux conseillères nationales ont interpellé le Conseil fédéral sur les coupures des budgets fédéraux d'APD et leurs conséquences. Mme Gurtner (Poch, BE) s'est élevée contre ces mesures et contre le peu de cas fait de la pétition des organismes d'entraide.

Mme Blunschy (PDC, SZ) regrettait les coupures faites dans I'APD, et que le Conseil national n'ait pas décidé de traiter de la pétition des œuvres d'entraide en même temps que du programme d'économies.

Rappelons que I'APD de la Confédération a été réduite pour 1984 de 50 millions de francs au titre des mesures d'assainissement proposées dans le rapport sur la planification financière 1984-1986 du 4 octobre 1982, et de 8 millions au titre des coupures linéaires, selon la décision du Conseil fédéral du 16 novembre 1983.

La fraction socialiste au Conseil national a déposé en juin 1983 un postulat demandant au Conseil fédéral d'examiner entre autres la possibilité d'accorder un soutien accru au Nicaragua dans le cadre de la coopération technique et de l'aide humanitaire de la Confédération.

Le Conseil fédéral s'est déclaré prêt à accepter le postulat dans le sens de I'octroi d'une aide humanitaire identique à celle qu'il accorde à d'autres pays.

M. Müller (parti évangélique, AG), conseiller national, a présenté en juin 1983 un postulat demandant à ce que soient explicités les critères qui servent à évaluer les projets d'aide au développement de la Confédération. Le Conseil fédéral a accepté le postulat, comptant présenter la réponse dans un rapport de gestion.

M. Ruffy (PS, VD)) a déposé le 16 décembre 1983 une interp̉ellation deman- 
dant au Conseil fédéral une aide accrue pour le Nordeste brésilien. Ce Conseil fédéral a répondu qu'un million de francs avaient été attribués au Nordeste; il s'est déclaré sensible à la détresse de cette région et prêt à examiner les demandes d'œuvres d'entraide sur le plan humanitaire.

Mme Blunschy (PDC, SZ) a déposé le 12 mars 1984 une interpellation proposant, face à la faim dans le monde, d'augmenter l'aide humanitaire de la Confédération. Mme Blunschy estime que les cas de I'Afrique et du Nordeste sont particulièrement préoccupants.

Le Conseil fédéral a répondu que de son point de vue la famine découle le plus souvent de carences dans l'infrastructure et dans la politique agricole. C'est pourquoi le Conseil fédéral préfère accorder une priorité à la coopération au développement. Néanmoins, un crédit spécial de 5 millions de francs a été attribué à l'Afrique, sous forme d'aide alimentaire. $54 \%$ de l'ensemble de cette forme d'aide va à l'Afrique.

Mme Friedli (PS, JU) a déposé le 19 mars 1984 une question ordinaire concernant le barrage de Manantali (Mali).

En novembre 1983, le Conseil fédéral a accordé la garantie des risques à l'exportation à l'entreprise Losinger, associée à un consortium international pour la construction d'un barrage sur le fleuve Sénégal, à Manantali au Mali, un des pays d'Afrique les moins avancés.

Selon une étude réalisée pour le compte du gouvernement américain, la construction de ce barrage provoquera le déplacement de 10 à 15.000 habitants sur des terres sans eau, donc inexploitables, et pourrait priver de leurs ressources traditionnelles de 100 à 300.000 autres qui vivent actuellement des crues du fleuve. La Banque mondiale a renoncé au financement de ce barrage qui est vivement critiqué par certains experts.

Mme Friedli demandait au Conseil fédéral si, pour respecter la loi sur la garantie contre les risques à l'exportation, le Conseil fédéral pouvait donner des précisions sur les détails de la consultation effectuée auprès des services concernés et si des mesures avaient été prises pour pallier les effets négatifs du projet (reboisement, planification de l'irrigation, etc.)

Le Conseil fédéral a répondu que la GRE ne couvre que la partie suisse du projet $(29 \%)$. La participation suisse se limite à l'exécution du barrage et il n'existe pas de possibilité d'influer sur le projet. Le financement était entièrement assuré au moment de la soumission. Le Conseil fédéral estime que seuls les principes fondamentaux de la politique suisse en matière d'aide au développement s'appliquent à la GRE. Du côté suisse, il n'est en principe pas prévu de soutenir des projets de développement dans la région du barrage. 
Commission consultative pour la coopération internationale au développement

Cette commission consultative a pour rôle principal de conseiller le Conseil fédéral en matière de coopération internationale au développement. Elle est composée de 17 membres choisis en dehors de l'administration fédérale : parlementaires et représentants des œuvres d'entraide, de l'économie privée, des syndicats, des universités et de la presse. Le secrétariat de la Commission est assuré par la section politique et recherche de la DDA. La Commission se réunit quatre fois par an.

En février 1983, la Commission a examiné l'esquisse d'un programme national de recherche sur les relations Suisse - Tiers Monde et a recommandé la sélection de ce thème en vue d'un financement par le Fonds national suisse de la recherche scientifique. Le Conseil fédéral n'a pas retenu de proposition de programme de recherche. La Commission s'est également penchée sur le nouveau crédit de programme prévu pour la participation de la Suisse au capital des banques régionales de développement.

La séance du mois de mai de la même année a été tout d'abord consacrée à la proposition de budget 1984 de l'aide publique au développement, puis à la préparation de la 6ème Conférence des Nations Unies sur le commerce et le développement (CNUCED VI). Le deuxième thème a été traité conjointement avec la Commission consultative de politique commerciale.

A l'ordre du jour de la réunion du mois de septembre figurait le programme de coopération de la DDA en Tanzanie. La Commission fut informée sur les possibilités d'action de la Suisse face à une situation socio-économique très difficile. La politique de concentration (géographique et sectorielle) de la DDA a ainsi pu être discutée à la lumière de cet exemple.

Lors de la dernière séance de l'année (novembre), les membres de la Commission se sont exprimés sur le Message concernant la continuation de la coopération technique et l'aide financière en faveur des pays en développement. Un plan détaillé avait été soumis à la Commission et de nombreuses suggestions émises au cours de la réunion ont pu être prises en considération au moment de la rédaction finale du Message.

La Commission est en outre tenue au courant des principales conférences et négociations internationales auxquelles participe la Suisse et est informée des faits marquants touchant à la politique de coopération au développement. 


\section{MESURES ECONOMIQUES ET COMMERCIALES}

Pour la première fois les mesures économiques et commerciales figurant au budget 1984 ont dépassé les 100 millions de francs. Leur part à l'aide publique au développement a quintuplé durant ces quatre dernières années. Le budget 1983 prévoyait encore 73,1 millions de francs, le montant 1984 est de 101,8 millions de francs grâce à l'adoption du premier paquet de mesures destinées à renforcer l'économie suisse.

Les fonds gérés par I'OFAEE ont pour but principal d'alimenter les aides à la balance des paiements et les crédits mixtes. Ces derniers constituent une aide liée qui ne peut être utilisée que pour se fournir en produits suisses. Une partie du crédit pouvant aller jusqu'à $50 \%$ est assurée par la Confédération selon des normes correspondant à l'aide publique au développement, le solde étant assumé par des banques commerciales.

Les crédits conclus actuellement le sont avec l'Egypte, le Honduras, I'Inde, le Kenya, le Cameroun, le Maroc, le Sénégal, le Sri-Lanka, la Thaïlande, la Tunisie et le Zimbabwe. Enfin des négociations sont engagées avec la Chine et I'Indonésie.

Un crédit mixte de vingt millions dont la Confédération fournit la moitié a été conclu avec la Banque ouest-africaine de développement le 22 juin 1984. C'est la première fois qu'un crédit mixte est accordé par la Suisse à une organisation internationale.

L'évaluation du premier crédit mixte attribué à I'Egypte a été réalisée en 1983. Les résultats de cette première évaluation ne sont pas publics.

\section{Etudes sur la promotion des importations}

Deux études sur les possibilités d'accroissement du volume des importations venant du Tiers Monde ont été menées en 1983. L'une grâce à un financement de I'OFAEE sur la distribution commerciale de produits en jute du Bangladesh, et l'autre pour le compte du comité d'action Pain pour le Pro: chain sur l'introduction possible d'un label certifiant la qualité du produit selon des normes de politique de développement.

Le jute est le principal produit d'exportation du Bangladesh (50\% des exportations). Mais la concurrence des produits synthétiques est sévère; la solution serait une amélioration de la productivité de l'industrie de transformation, une promotion générale des ventes et la conclusion d'accords bilatéraux sur les prix. Les perspectives sur le marché suisse ne sont pas excellentes et dépendent beaucoup de la mode et des produits proposés selon leur finition, leur forme, etc. Quoique le chiffre d'affaires ne puisse être très important, les incidences pourraient être déterminantes pour certains producteurs du Bangladesh. D'autre part il est possible d'étendre de façon importante le réseau de distribution. 
Quant au label de qualité propre aux produits du Tiers Monde, une telle marque distinctive ne saurait être introduite avant que la provenance des produits ne soit obligatoirement mentionnée et avant qu'un nombre d'articles suffisant puisse être proposé, ce qui est difficile vu les contrôles à effectuer quant aux conditions de travail et de production.

\section{Aide à la balance des paiements}

De juillet 1983 à juin 1984, trois pays africains, le Ghana, Madagascar et le Soudan, ont bénéficié d'aides suisses à la balance des paiements. Les deux derniers pays avaient déjà obtenu de telles contributions auparavant.

Deux accords ont été conclus avec le Soudan, en juillet 1983 et en mai 1984, portant chacun sur un montant de 10 millions de francs. Le premier accord prévoit le financement d'importations de médicaments de base ainsi que de matières premières, de produits semi-finis et de pièces de rechange pour l'industrie et l'agriculture. II s'agit d'aider le Soudan à mieux utiliser ses capacités de production et à améliorer I'approvisionnement en produits pharmaceutiques.

Le deuxième accord fait partie d'un programme d'aide financé principalement par I'Agence internationale du développement (AID). Le programme a pour but de soutenir le secteur agricole irrigué en vue d'accroître la production de coton. Le coût du programme s'élève à 90 millions de dollars; 50 millions sont assurés par I'AID.

Un second accord d'aide à la balance des paiements a été conclu le 12 janvier 1984 avec Madagascar. Un crédit de 10 millions de francs servira au financement de matières premières, de pièces de rechange, de produits semi-finis et d'outils pour des.secteurs-clef de l'économie malgache. L'aide devrait permettre d'accroître la production de riz et de mieux utiliser les capacités de production existantes dans les domaines artisanal et industriel. Le montant de l'aide a été prélevé sur le crédit complémentaire concernant des "mesures destinées à renforcer l'économie suisse" décidées par le Conseil fédéral au début de 1983.

L'aide destinée à soutenir la balance des paiements du Ghana s'inscrit également dans le programmẻ de relance de l'économie suisse. Son montant de 12,7 millions de francs est prévu pour la remise en état du parc roulant de la Société des transports publics (State Transport Corporation) du Ghana. Plus des deux tiers des 190 poids lourds et camions-citernes (de marque Saurer) n'étaient plus en état de marche en raison du manque de pièces de rechange.

L'aide octroyée au Ghana servira à l'achat des pièces de rechange nécessaires et d'autres matériels. Elle permettra de financer également les travaux de réparation et d'entretien qui seront effectués par la Société Saurer, ainsi que le perfectionnement des conducteurs et des mécaniciens.

Bien que les aides à la balance des paiements n'aient, en majeure partie, pas été liées à l'achat de biens suisses, les crédits ont néanmoins été surtout 
dépensés en Suisse. En 1983, le Soudan a consacré près des trois quarts de I'aide obtenue (10 millions de francs) à l'acquisition de fournitures suisses. Sur le premier crédit, d'un montant de 10 millions de francs également, accordé en 1982 à Madagascar, près deux deux tiers ont été dépensés en Suisse. Le deuxième accord d'aide à la balance des paiements malgache entraînera une proportion encore plus grande d'achats en Suisse. Quant à l'aide accordée au Ghana, on prévoit que la proportion des dépenses effectuées en Suisse sera plus importante encore.

\section{Sources}

EVD-Pressemitteilungen, 20.7.83, 16.5.84.

EVD-Pressemitteilung, 16.1.84.

NZZ, 14.4.84.

\section{COOPERATION TECHNIQUE ET AIDE FINANCIERE}

Les dépenses encourues pour l'aide technique bilatérale fournie en 1983 ont émargé au crédit de programme de 1.650 millions de francs accordé par le Parlement en 1980. Les accents principaux sont restés placés sur le développement agricole avec $27 \%$ des dépenses ventilées et l'éducation avec $11 \%$. Les banques, le commerce et le tourisme ont reçu $11 \%$ des moyens, l'industrie et l'artisanat $9 \%$, la santé $9 \%$ également.

L'actuel crédit de programme devrait être épuisé au 31 octobre 1984 pour céder la place au nouveau crédit de 1.800 millions de francs, lequel aura une durée d'au moins trois ans.

Au cours de l'année 1983, la DDA a eu la responsabilité de 514 projets en cours (464 projets d'assistance technique dont $\mathbf{4 6}$ actions nouvelles et 50 projets d'aide financière dont 29 nouvelles actions).

L'effectif du personnel octroyé à l'administration de la DDA est demeuré inchangé en 1983; 143 des 144,5 postes de travail autorisés étaient occupés. Par contre le nombre des collaborateurs engagés pour des tâches à l'étranger sous contrat de droit privé a passé de 343 en 1982 à 290 en 1983. Ces chiffres ne prennent pas en compte le personnel engagé dans des projets réalisés sous mandat de la DDA mais dont elle conserve la responsabilité. Deux causes principales à cette réduction du nombre de coopérants : le transfert à d'autres organisations de projets de la DDA et l'allongement de la durée des contrats, qui diminue le nombre de collaborateurs occupant temporairement le même poste. 


\subsection{Coopération bilatérale au développement}

La coopération au développement bilatérale de la Confédération s'est montée en 1983 à 287,2 millions de francs dont 107,5 millions à la coopération technique. Les actions réalisées directement par la DDA en 1983 dans ces domaines (coopération technique et aide financière confondues) ont représenté $43,9 \%$ du volume total des dépenses. $19,7 \%$ des 287,2 millions de francs (1982 : 15,5\%) ont été confiés à des institutions et entreprises suisses pour l'exécution en régie de ces actions.

\section{REPARTITION GEOGRAPHIQUE DES ACTIONS DE COOPERATION TECHNIQUE ET D'AIDE FINANCIERE BILATERALES DE LA DDA EN 1983}

(Versements en millions de francs et en pourcentages)

\begin{tabular}{lrr} 
Région & Mio Fr. & $\%$ \\
\hline Afrique & 118,1 & 41,1 \\
Amérique latine & 52,0 & 18,1 \\
Asie & 95,3 & 33,2 \\
Europe & 1,0 & 0,3 \\
Non classé & 20,8 & 7,3 \\
\cline { 2 - 3 } TOTAL & $\mathbf{2 8 7 , 2}$ & $\mathbf{1 0 0 , 0}$ \\
\hline
\end{tabular}

Note: A l'intérieur du groupe des pays les plus pauvres, le plus gros de l'aide s'est adressé à 18 pays et une région dite de concentration qui, à eux seul, ont bénéficié de $60 \%$ de notre effort bilatéral.

Collaboration avec les œuvres d'entraide privées suisses

Un certain nombre d'œuvires d'entraide suisses exercent une activité de coopération au développement depuis plus longtemps que la DDA. Elles ont réalisé de nombreux projets dans des pays en développement et ont ainsi acquis une vaste expérience. En tant qu'organisations privées, elles peuvent, quelquefois mieux que des institutions publiques, atteindre les couches marginales de la population et tisser des liens étroits avec des organisations non-gouvernementales des pays en développement.

La collaboration avec les œuvres d'entraide privées peut revêtir deux formes : les contrats de régie par lesquels la DDA leur délègue la réalisation de projets qu'elle a identifiés et élaborés; les contributions de la Confédération aux 
projets des œuvres d'entraide, contributions qui couvrent au maximum les deux tiers du budget total du projet. Outre le financement de projets spécifiques, la DDA a ouvert à six d'entre elles - Helvetas, Swissaid, Action de Carême, Pain pour le prochain, Caritas, Entraide protestante suisse - une ligne de crédit pour un ensemble de projets d'une durée d'une ou plusieurs années.

La DDA a, en 1983, versé aux œuvres d'entraide un montant global de 70,5 millions de francs, répartis entre quelque $\mathbf{4 0}$ organisations et 167 projets de développement. Sur ce montant, 27,3 millions de francs ont permis à la DDA de soutenir des actions définies et réalisées par les œuvres d'entraide et 43,2 millions de francs ont été utilisés pour financer l'exécution des projets en régie. Le volume des mandats confiés en régie à Intercoopération a atteint 23 millions de francs.

\section{Collaboration avec l'économie privée suisse}

L'intérêt que la DDA porte à une collaboration plus intense avec l'économie privée s'est manifesté en 1983 par la poursuite des contacts directs en vue d'échanger des informations tant de caractère général que liées à l'exécution d'actions spécifiques. Le caractère délié de l'aide suisse n'empêche pas la DDA de faire souvent appel aux prestations de l'économie privée, que ce soit par le biais de commandes de matériel passées à des entreprises suisses ou par le biais de mandats confiés à des bureaux d'ingénieurs ou des formes de consultants. Ces mandats peuvent se limiter à certains volets techniques à I'intérieur de projets (expertises, évaluations, conseils, etc.) ou peuvent être confiés pour la réalisation globale de projets (projets en régie).

La DDA a conclu en 1983 : 183 contrats avec des experts individuels pour des missions de courte durée (représentant au total 1,5 million de francs) et 27 contrats avec des firmes de consultants ou bureaux d'ingénieurs conseil pour des études, des évaluations ou des missions (pour un montant global de 2,5 millions de francs).

Plusieurs projets de coopération technique, présentant des caractéristiques très particulières, sont en outre exécutés en régie par des firmes privées : il s'agit principalement de projets dans les domaines de I'hôtellerie, l'énergie et la production laitière. (Les nouveaux contrats signés en 1983 ont représenté 5,1 millions de francs, alors que les dépenses se sont élevées à 2,6 millions de francs.)

A l'intérieur du groupe des pays les plus pauvres, le plus gros de l'aide s'est adressé à 18 pays et une région dits de concentration qui, à eux seuls, ont bénéficié de $60 \%$ de notre effort bilatéral. 
REPARTITION DES ACTIONS DE COOPERATION TECHNIQUE ET D'AIDE

FINANCIERE BILATERALES DE LA DDA SELON LE NIVEAU DE REVENU DES PAYS BENEFICIAIRES EN 1983

(Versements en millions de francs et en pourcentages)

\begin{tabular}{lcccc} 
Groupes de pays (1) & $\begin{array}{c}\text { Coopération } \\
\text { technique } \\
\text { Mio fr. }\end{array}$ & $\begin{array}{c}\text { Aide } \\
\text { financière } \\
\text { Mio fr. }\end{array}$ & Mio fr. & $\%$ \\
\hline $\begin{array}{l}\text { Pays les moins } \\
\text { avancés (PMA) }\end{array}$ & 84,6 & 21,0 & 105,6 & 47,5 \\
$\begin{array}{l}\text { Autres pays à faible } \\
\text { revenu (PNB/hab. 600 \$) }\end{array}$ & 50,2 & 41,2 & 91,4 & 41,1 \\
$\begin{array}{l}\text { Pays à revenu intermé- } \\
\text { diaire (PNB/hab. 600 \$) }\end{array}$ & 23,0 & 2,4 & 25,4 & 11,4 \\
\cline { 2 - 5 } Total aide ventilée & 157,8 & 64,6 & 222,4 & 100,0 \\
\hline $\begin{array}{l}\text { Projets régionaux } \\
\text { et non classé (2) }\end{array}$ & 49,7 & 15,1 & 64,8 & \\
\cline { 2 - 5 } TOTAL & $\mathbf{2 0 7 , 5}$ & $\mathbf{7 9 , 7}$ & $\mathbf{2 8 7 , 2}$ & \\
\hline
\end{tabular}

1. Les groupes de pays ont été établis selon la définition adoptée par l'OCDE et se réfèrent au produit national brut par habitant de 1980.

2. Opérations ne pouvant être ventilées géographiquement telles que contributions à des cours, à des programmes, crédits globaux pour les boursiers, financement de recherches, soutien au programme de certaines organisations, etc.

\section{Aide associée et cofinancement}

La collaboration que la DDA entretient avec les organismes multilatéraux pour l'exécution de projets spécifiques reçoit le nom d'aide associée lorsqu'il s'agit de projets d'assistance technique gérés par des institutions spécialisées du système des Nafions Unies et de cofinancement pour des projets d'aide financière réalisés par des banques de fonds de développement. Dans un cas comme dans l'autre, la procédure est la même : la DDA sélectionne un projet particulier à l'intérieur du programme de l'organisation internationale. Très souvent, elle participe directement à la formulation du projet et aux négociations avec le pays bénéficiaire. La DDA a la possibilité de participer également à des missions de supervision ou d'évaluation. Dans le cas des projets d'aide associée, la totalité du financement externe du projet, y compris les frais d'agence, est prise en charge par la DDA. Pour les cofinancements, comme leur nom l'indique, le financement est assuré par diverses sources : la banque ou le fonds lui-même et un ou plusieurs 
donateurs bilatéraux ou multilatéraux. Cette forme de collaboration permet de mobiliser les connaissances approfondies dont disposent les organismes internationaux au service de projets répondant de près aux objectifs de l'aide suisse.

Les chiffres concernant les actions d'aide associée et le confinancement se trouvent dans la partie statistique.

\section{Appui de la DDA aux travaux d'instituts de recherche internationaux}

La DDA octroie, depuis plusieurs années, des contributions à certains instituts de recherche internationaux d'intérêt pour les pays en développement. Elle soutient en particulier les centres régionaux dépendant du Groupe consultatif pour la recherche agricole internationale (CGIAR). Actuellement dix centres bénéficient de l'appui de la Suisse, que ce soit par des contributions globales ou par le financement de programmes particuliers. 18 millions de francs ont ainsi été versés à ce groupe en 1983.

D'autres instituts de recherche de dimension internationale bénéficient du soutien de la Suisse. II s'agit d'instituts spécialisés dans la recherche médicale (maladies infectieuses, tuberculose), la protection de l'environnement ou la recherche agro-forestière. Le détail des versements effectués en 1983 en faveur de ces instituts figure au tableau de la page 102.

Soutien de la DDA à certaines institutions universitaires suisses et participation à leurs activités en 1983

La DDA a adopté en 1983 de nouvelles lignes directrices internes sur les relations avec les universités et institutions universitaires suisses. Une des conséquences pratiques immédiates a été la mise sur pied d'un petit programme d'encouragement aux contacts entre institutions universitaires suisses et dans le Tiers Monde. La décision a été prise en 1984 de relancer le programme des jeunes chercheurs de la DDA.

L'appui a été poursuivi aux écoles polytechniques fédérales de Lausanne et de Zurich pour leurs cours postgrades respectifs sur le développement. Des contacts ont été noués pour des mandats et des appuis techniques aux instituts spécialisés de I'EPFZ suivants : Institut des sciences alimentaires; Institut de production animale; Institut d'économie rurale; Institut pour l'aménagement, l'épuration et la protection des eaux.

\subsection{Coopération multilatérale}

La coopération au développement multilatérale fait partie intégrante des efforts entrepris par la communauté internationale pour venir en aide au Tiers Monde. La Suisse y participe. 


\section{APPUI DE LA DDA AUX TRAVAUX D'INSTITUTS DE RECHERCHE INTERNATIONAUX EN 1983}

(Versements nets en millions de francs)

Contributions
ordinaires $\begin{gathered}\text { Projets et } \\ \text { programmes } \\ \text { particuliers }\end{gathered}$

Centres de recherche du Groupe consultatif pour la recherche agricole internationale (CGIAR)

maïs et du blé (CIMMYT), Mexique

Institut international de recherches sur les cultures des zones tropicales semi-arides

(ICRISAT), Inde

Centre international pour l'élevage en

Afrique (ILCA), Ethiopie

Centre international de la pomme de terre (CIP), Pérou

Laboratoire international de recherche sur les maladies des animaux (ILRAD), Kenya

Association pour le développement de la riziculture en Afrique de I'Ouest (WARDA), Libéria

Autres centres

Autres instituts de recherche

Institut international d'agriculture

tropicale (IITA), Nigéria

International Centre for Diarrhoeal Diseases

Research (ICDDR), Bangladesh

Programme de recherche et de formation

sur les questions liées à l'environnement

(ENDA), Sénégal

Union internationale contre la tuberculose

(UICT)

International Council for Research in

Agro-Forestry (ICRAF), Kenya

Autres instituts

TOTAL
13,5

2,7

3,0

4,8

18,3

2,8

5,5

3,5

2,6

0,5

2,6

2,1

2,1

1,0

1,1

2,1

1,2

1,2

$-$

4,0

4,0

0,8

1,2

1,8

4,5

6,3

$-$

2,7

2,7

1,3

1,3

-

0,8

0,8

$-$

0,7

0,7

-

0,7

0,5

$-$

0,5

$-$

0,3

0,3

15,3

9,3 
L'aide multilatérale, de l'avis du Conseil fédéral, exerce des fonctions complémentaires que l'aide bilatérale ne peut pas remplir. L'aide multilatérale est constante, alors que l'aide bilatérale dépend souvent de facteurs conjoncturels et à court terme.

Les institutions de la coopération multilatérale ont souvent une dimension et une infrastructure qui leur permettent de maîtriser les nouveaux problèmes et de jouer un rôle de coordinateur. Certaines actions doivent être menées à très grande échelle.

Les organisations spécialisées ont l'expérience et les connaissances nécessaires dans leur domaine. La dimension des organisations et de leurs projets leur permet de faire appel à des capitaux privés pour des cofinancements.

Les organisations actives dans la coopération multilatérale jouent un rôle de coordination entre les pays pour définir les stratégies de développement nationales et internationales. L'aide multilatérale n'est pas liée, ce qui représente un avantage pour les pays en voie de développement.

En ce qui concerne les compétences au sein de l'administration fédérale, la DDA est responsable en priorité de ce qui touche à l'assistance technique. Elle partage avec I'OFAEE la responsabilité de l'aide financière. Dans le cas des organisations internationales qui sont du ressort d'autres services ou 'départements, la DDA est appelée à donner son avis sur les questions relevant de la politique de développement.

Depuis 1982 la Suisse utilise le mode de paiement des "promisory-notes" dans le domaine de l'aide financière internationale. II s'agit de contributions effectuées sous la forme de reconnaissances de dette irrévocables mais qui ne sont comptabilisées qu'au moment des paiements effectifs du fait que ceux-ci peuvent s'échelonner sur près d'une décennie. La phase de transition pourrait durer quelques années encore.

Par la suite, les deux catégories de contributions bilatérales et multilatérales devraient retrouver un équilibre dans les proportions que nous lui connaissons, c'est-à-dire approximativement $40 \%$ pour le multilatéral et $60 \%$ pour le bilatéral.

Comme les années précédentes, la contribution la plus importante de la Suisse sur le plan multilatéral est allée au Programme des Nations Unies pour le développement (PNUD). Le PNUD joue un rôle majeur dans la coopération internationale. Le PNUD ouvre en faveur de chaque pays en développement un crédit-cadre qui permettra de réaliser un programme répondant le mieux possible aux besoins des destinataires de l'aide. $80 \%$ environ des ressources du PNUD vont aux pays les plus pauvres. La Suisse verse au PNUD 2,5\% de l'ensemble des versements. Par ailleurs, la Suisse effectue des versements auprès des organisations suivantes : 
Le Fonds pour les pays les moins avancés (FPMA). II s'agit d'un Fonds spécial créé au sein du PNUD et dont l'insertion dans le programme ordinaire de ce dernier assure une utilisation optimale des moyens. En 1983, la Suisse a versé au Fonds pour les PMA une contribution ordinaire de 4,84 millions. Par ailleurs, cette même année, le Fonds a reçu de la Suisse une contribution extraordinaire de 5 millions dans le cadre d'une aide financière à court terme en faveur du PNUD.

Le Fonds d'équipement des Nations Unies (FENU). Ce Fonds, dont la création remonte à 1966, bénéficie aujourd'hui presque exclusivement aux pays les moins avancés. II a notamment pour mandat de fournir aux PMA une aide en équipement pour des projets de petites et moyennes dimensions. II veille à ce que les projets qu'il finance contribuent à satisfaire les besoins vitaux des groupes les plus déshérités de la population. Les ressources du FENU proviennent d'un nombre limité de pays dont la Suisse.

Le Fonds des Nations Unies pour les activités en matière de population (FNUAP). Le FNUAP est aujourd'hui la principale institution internationale finançant et exerçant des activités en matière de population. Son financement est assuré par les contributions volontaires annuelles des pays membres. L'exécution de ses projets est, en règle générale, confiée aux institutions spécialisées des Nations Unies telles que I'OMS, I'UNICEF, I'UNESCO ou aux gouvernements de pays intéressés eux-mêmes.

Le Fonds des Nations Unies pour I'enfance (UNICEF). Dès I'origine, la Suisse a été directement associée aux activités de I'UNICEF. En effet, depuis la création du Fonds, notre pays a occupé de façon ininterrompue un siège à son Conseil d'administration.

Organisation mondiale de la santé (OMS). Dans le cadre de la XXXVle Assemblée générale de I'Organisation, la Suisse a notamment participé aux travaux visant à réviser et à compléter la stratégie mise au point sous le titre de "Santé pour tous d'ici l'an 2000". Par ailleurs, la DDA a soutenu en 1983 deux actions de l'OMS à concurrence d'un montant total de 2,25 millions - la lutte contre les maladies diarrhéiques et le programme de recherche et de formation contre les maladies tropicales.

Organisation des Nations Unies pour l'éducation, la science et la culture (UNESCO). La DDA s'est engagée en 1983 à soutenir un projet de formation dans le domaine des télécommunications au Zimbabwe pour un montant de 2,9 millions au titre de l'aide associée.

Fonds international de développement agricole (FIDA). Le FIDA a été créé en 1977 dans le but de mobiliser des ressources financières supplémentaires qui aideraient les pays en développement à augmenter la production vivrière indigène et à améliorer les bases nutritionnelles des populations.

Les membres de la FIDA sont groupés en trois catégories qui disposent, indépendamment de leur contribution ou de leur effectif, du même nombre 
de vois : pays industrialisés, pays de I'OPEP, pays en développement.

Quant au financement, il est essentiellement opéré par les membres des deux premiers groupes et ceci dans la proportion actuelle de $58 \%$ pour les pays industrialisés et de $\mathbf{4 2} \%$ pour les Etats pétroliers.

La situation du Fonds est relativement précaire après que les Etats-Unis eurent annoncé en décembre 1983 que, au lieu de la contribution de 183 millions de dollars qu'ils devaient verser jusqu'à la fin de l'année 1983, ils n'en fourniraient que la moitié, donc 90 millions de dollars.

Ainsi dans le groupe I seuls la Suisse, I'Australie, les Pays-Bas, I'Autriche, I'Italie et les pays scandinaves ont versé la totalité de leur contribution, les autres pays n'ont pas voulu prendre de décision avant de connaître la position définitive des Etats-Unis.

L'attitude des Etats-Unis pourrait s'expliquer par leur déception de voir les pays de I'OPEP couvrir une proportion moins large que prévu des dépenses.

A l'occasion de la Conférence annuelle des Nations Unies qui s'est tenue à New York, l'observateur permanent de la Suisse auprès de I'ONU, Mme I'ambassadeur Francesca Pommetta a annoncé les principales contributions que la Suisse entend faire à différentes institutions, Fonds et programmes spéciaux du système des Nations Unies.

La plus importante des contributions suisses - à savoir $\mathbf{4 0}$ millions de francs suisses - ira, comme d'habitude, au Programme des Nations Unies pour le développement qui constitue l'organe de financement et de coordination central de I'ONU pour la coopération technique.

Le Fonds des Nations Unies pour I'enfance (UNICEF) recevra 9 millions de francs en tant que contribution générale. La Suisse participera en outre au financement de plusieurs projets spécifiques.

Les autres contributions importantes sont destinées aux Fonds et programmes spéciaux suivants : 5,3 millions de francs au Fonds pour les pays les moins avancés; 4,235 millions au Fonds d'équipement; 4 millions au Fonds pour les activités en matière de population (FNUAP) et 0,4 million au Programme des volontaires.

La Suisse a en outre réservé pour deux ans une somme de 4,5 millions pour le financement de projets du Fonds pour le développement industriel (FNUDI) et fera une contribution de 4 millions de francs pour les années 1983-1986 au Programme conjoint du PNUD et de la Banque mondiale en matière d'énergie.

En tant que membre des organes de direction du PNUD et de I'UNICEF, la Suisse participe activement à la conception et au contrôle des activités de ces institutions. 
Banques et Fonds régionaux de développement

Le Conseil fédéral a décidé de participer à la sixième augmentation du capital de la Banque interaméricaine de développement et de porter la part suisse à 65 millions de francs. Les $4,5 \%$ de ce montant (2,95 millions de francs) seront effectivement versés. Le reste constituera un capital de garantie. La Suisse participe également à la sixième reconstitution du Fonds des opérations spéciales rattaché à la Banque, avec un don d'environ 11,5 millions de francs suisses.

Les montants mentionnés, payables en quatre tranches annuelles (19831986), sont imputés à des crédits de programmes déjà existants. Le Parlement avait déjà, à l'époque, approuvé l'utilisation prévue de ces crédits. Depuis 1979, la Suisse est membre de la Banque interaméricaine de développement.

La Banque de Développement des Etats de l'Afrique centrale (BDEAC) et le gouvernement suisse ont signé le 17 février 1984 un accord portant sur I'octroi à cette institution d'une aide financière de 6,8 millions de francs destinée à améliorer les conditions d'existence des populations et zones défavorisées de la région d'Afrique centrale.

La BDEAC est une Banque de développement sous-régionale créée en 1975 par le Cameroun, le Congo, le Gabon, la République centrafricaine et le Tchad. Objectif poursuivi : développer les efforts de coopération afin de réduire les disparités régionales en contribuant à combler notamment le retard du secteur agricole.

Depuis 1980 , la DDA s'est attachée à aider la BDEAC à mettre sur pied une division du développement rural. Aujourd'hui cette structure est opérationnelle. Elle se trouve en mesure d'identifier, de mettre au point et de suivre des projets de développement rural dans la région, en s'assurant au besoin les services de consultants (agronomes, ingénieurs en génie civil, économistes, etc.).

L'aide financière accordée par la Suisse à la BDEAC complète les appuis déjà consentis à cette institution. Elle comporte deux volets : une contribution non remboursable de Fr. 800.000.- destinée à renforcer encore la capacité de la Banque dans le domaine du développement rural (les fonds suisses permettront de financer des études de préparation de projets et des missions de consultants); un prêt de Fr. 6.000.000.- à des conditions de faveur pour le cofinancement avec la Banque de projets de développement rural.

Les engagements de l'aide financière suisse se feront sur une période d'environ deux ans. Dans le cadre de l'accord signé avec la Banque, la DDA contrôlera l'affectation et l'utilisation des fonds suisses. 


\section{L'AIDE HUMANITAIRE}

Le total des dépenses pour l'aide humanitaire de la Confédération s'est élevé en 1983 à 124,94 millions de francs. Ce total comprend les dépenses en faveur de pays qui ne sont pas officiellement considérés comme pays en développement. Ainsi, en 1983, 1,6 million de francs, en 1982, 3,9 millions de francs ont été utilisés en dehors du Tiers Monde.

A côté des interventions dans les cas de catastrophes naturelles et de conflits armés, la part de l'assistance aux réfugiés et aux personnes déplacées va croissant.

L'aide humanitaire est gérée en coopération avec les organisations d'entraide nationales et internationales et en collaboration avec les volontaires du Corps d'aide en cas de catastrophes.

L'année 1983 a vu s'élargir le champ d'intervention du Corps d'aide en cas de catastrophes. 145 volontaires ont été engagés dans les actions de secours. Le Corps disposait à la fin de l'année d'un stock de matériel d'intervention d'une valeur de 4,5 millions de francs, alors que les dépenses s'élevaient à quelque 2,2 millions, soit un quart environ du débours total.

Des recherches ont été menées à bon terme en vue de l'acquisition de nouveaux équipements hydrauliques mobiles, ainsi que pour I'amélioration de tentes familiales à double toit et cloisons hautes.

Le Corps d'aide en cas de catastrophes est intervenu en 1983 en Guinée équatoriale, au Ghana, en Guinée-Conakry, en Italie, en Colombie, au Yemen du Nord, en Haute-Volta, au Pérou, au Yemen du Sud, au Tchad et en Turquie.

La répartition par région de l'aide humanitaire donne les proportions suivantes : Afrique 53,5\%; Moyen-Orient 13,2\%; Asie 18,1\%; Amérique latine 9,1\%; Europe $1,1 \%$; divers $5 \%$.

En ce qui concerne l'aide alimentaire, 36.400 tonnes de denrées d'une valeur globale de 55,6 millions de francs (financement, transport et livraison compris) ont été livrées par la Suisse en 1983. Ce total représentait 0,4\% de I'aide alimentaire mondiale.

$85 \%$ de cette aide étaient destinés à des réfugiés et des personnes en détresse; $60 \%$ ont été distribués en Afrique.

$51 \%$ de l'aide céréalière provenaient des pays en développement eux-mêmes (en Afrique surtout).

L'Inde, le Tchad, I'Ethiopie, le Liban, le Malawi, le Brésil, les réfugiés palestiniens au Proche-Orient et les réfugiès de I'Ogaden en Somalie ont été les principaux bénéficiaires de l'aide en produits laitiers. 
L'aide céréalière visait surtout quant à elle les réfugiés palestiniens, l'Ethiopie, le Ghana et le Rwanda. 2,7 millions de francs ont été confiés à des œuvres d'entraide suisses pour la réalisation d'actions humanitaires d'urgence dans 19 pays. Les actions de secours pour un montant de un million de francs ont également été réalisées avec le concours de neuf représentations suisses.

La Confédération a annoncé qu'elle verserait au HCR en 1984 une contribution ordinaire de 4,5 millions de francs et octroierait dans la mesure des possibilités financières des contributions extraordinaires en faveur de programmes spéciaux, et enfin mettrait à disposition pour des missions de durée limitée des experts du Corps suisse pour l'aide en cas de catastrophes à l'étranger. Ces contributions seront imputées au crédit de programme pour l'aide humanitaire internationale. La Suisse est membre du comité exécutif du HCR.

Le Conseil fédéral a décidé d'accroître l'aide alimentaire d'urgence de cinq millions de francs en faveur de I'Afrique en 1984. Cette contribution extraordinaire va à la charge du crédit de programme de l'aide humanitaire internationale.

Les pays les plus menacés actuellement en Afrique sont : I'Ethiopie, le Ghana, la Guinée-Bissau, le Cap-Vert et le Mozambique.

Durant l'année écoulée, l'aide en céréales de la Confédération s'est montée à 24.130 tonnes (29.405 tonnes d'équivalent de blé). Elle comprenait les produits suivants : 10.500 tonnes de farine panifiable suisse ainsi que 13.630 tonnes de maïs, farine de maïs et de sorgho provenant d'Argentine, du Kenya et du Rwanda.

La marchandise a été acheminée en Bolivie, Ethiopie, Ghana, Mali, Pérou, Rwanda et au Moyen-Orient pour des opérations de secours de Caritas, du CICR, du Programme alimentaire mondial et de I'UNRWA. En outre une attribution a été faite au gouvernement du Cap-Vert.

L'aide en céréales de la Suisse s'inscrit dans le contexte de la Convention relative à l'aide alimentaire issue de l'Accord international sur le blé, dont elle est membre.

L'engagement minimal par an est de 27.000 tonnes d'équivalent de blé. Ce tonnage correspond à $0,35 \%$ de tous les engagements de 7,6 millions de tonnes au total pris par les douze membres.

Dans le cadre de cette aide humanitaire internationale, il est prévu de livrer en 198427.000 tonnes de céréales à des pays en développement, principalement à titre d'aide d'urgence et d'aide aux réfugiés. Le crédit disponible à cet effet s'élève selon le budget à 17,85 millions de francs. 


\section{BOURSES}

La modification apportée à l'arrêté fédéral du 19 décembre 1980 a continué $d^{\prime}$ 'assurer le financement du programme de bourses universitaires que gère I'Office fédéral des affaires culturelles du DFI; une majorité des étudiants poursuivant leurs études en Suisse proviennent de pays du Tiers Monde (180 étudiants). Les dépenses du DFI se sont élevées à 2,5 millions de francs.

De plus, dans le cadre de la coopération technique bilatérale (aide associée comprise), 284 boursiers ont été formés en Suisse en 1983. Ces stages de perfectionnement s'adressent à des ressortissants du Tiers Monde ayant déjà une expérience professionnelle. Les dépenses y relatives se sont élevées à 3 millions de francs environ. $65 \%$ d'entre eux provenaient d'Afrique et $19 \%$ d'Asie. La plupart des boursiers ont reçu une formation dans les domaines techniques et professionnels (17\%), des services (postes et télécommunications, hôtellerie, santé publique, respectivement $13 \%, 10 \%$ et $10 \%)$; assurances $(6 \%)$, banque $(4 \%)$, ou de l'administration publique $(6 \%)$.

\section{PRESTATIONS DES CANTONS ET DES COMMUNES}

Ces collectivités publiques ont fourni en 1983 des prestations d'un montant total de 8,42 millions de francs représentant quelque $1,5 \%$ du total de I'APD de la Suisse.

Vingt-deux cantons ont fourni environ 4 millions et 140 communes 4,5 millions. Près de $80 \%$ de ces contributions relèvent de la coopération au développement, $20 \%$ relevant de l'aide humanitaire.

Des 4 millions de francs déboursés par les cantons, $73 \%$ I'ont été par les 4 cantons de Genève, Bâle-Ville, Zoug et Zurich. Des 4,5 millions de francs de prestations d'APD des communes, plus de $77 \%$ proviennent de 65 communes situées dans les cantons de Zurich, Genève et Berne.

\section{LA COOPERATION AU DEVELOPPEMENT DES ORGANISATIONS PRIVEES}

La coopération privée au développement constitue une prestation autonome des différents organismes d'entraide, des œuvres missionnaires et d'autres organisations privées en Suisse. Leurs activités sont en partie liées financièrement et organisées en collaboration avec la Confédération; en particulier, cer- 
taines prestations privées sont complétées par des fonds de la Confédération. En 1983, les organisations privées au développement à caractère religieux ou non ont pu consacrer 125,17 millions de francs de dons à leurs activités d'aide au développement. En 1982, ce montant s'est élevé à 105,4 millions de francs. Ces activités se divisent entre l'aide au développement proprement dite, pour laquelle 103,8 millions de francs étaient à disposition, et l'aide humanitaire, pour laquelle 21,78 millions de francs étaient disponibles. L'aide des organisations privées au développement a été complétée par des fonds de la Confédération d'un montant de 373,72 millions de francs; I'aide humanitaire des organisations privées a reçu quant à elle un complément fédéral se montant à 123,36 millions de francs.

Ce sont les œuvres d'entraide Caritas, EPER, Action de Carême, Croix-Rouge suisse et les œuvres missionnaires "Institut Ingenbohl" et "Bethlehem" qui ont pu rassembler le plus de fonds. Intercoopération et Helvetas ont reçu le plus de contributions fédérales.

L'action des organismes privés d'entraide ne se limite pas à la coopération technique et à l'aide humanitaire. Nombreuses sont les activités en Suisse même. Certaines organisations ne gèrent aucun projet dans les pays en développement. Ces dernières voient leur but principal dans l'information du public au sujet des questions de développement.

L'activité publique la plus importante menée durant l'année en revue par les œuvres d'entraide a été l'action "Pour un monde sans famine" des organismes Swissaid, Action de Carême, Pain pour le Prochain, Helvetas, EPER, Caritas, Entraide ouvrière suisse et la Croix-Rouge suisse. Ces derniers ont lancé, en mai 1984, cette campagne d'information et de collecte de fonds, avec la collaboration de la "Chaîne du bonheur" de la radio et de la télévision. Jusqu'à l'été 1984, 10 millions de francs ont été récoltés dans le cadre de cette campagne. L'action se poursuivra jusqu'à la fin de 1984 et a pour but d'informer sur les diverses causes de la faim, de permettre des mesures d'aide d'urgence à court terme dans les pays les plus durement touchés par la faim en Afrique et en Amérique latine et enfin, de renforcer le travail à plus long terme dans les projets des œuvres d'entraide.

L'action de l'année précédente, "aider pour survivre demain", avait rassemblé les œuvres d'entraide, qui avaient donné plus de poids à cette action par le biais d'une pétition. En mai 1984, celles-ci se sont à nouveau tournées vers I'opinion publique avec un sondage d'opinion sur la politique de développement (voir aussi Annuaire 1984, Analyses et positions). Elles jugent les résultats de ce sondage, lequel a été réalisé pour le compte commun de la DDA et des œuvres d'entraide, très encourageants pour leur travail, mais doivent toujours déplorer le manque d'information parmi le public suisse sur les pays en voie de développement et sur la coopération au développement.

En même temps, les œuvres d'entraide ont invité les parlementaires à renoncer aux mesures d'économies et aux coupures prévues dans l'aide au déve- 
loppement. Elles estiment que l'aide future de la Suisse devrait être de plus en plus orientée vers les buts suivants :

- adaptation de l'aide aux besoins fondamentaux des couches les plus pauvres de la population et choix d'organisations non-gouvernementales comme partenaires pour les projets,

- encouragement redoublé de l'artisanat et des petites entreprises afin de régler le problème de l'emploi,

- mesures de protection de l'environnement et combat contre l'explosion démographique.

La coopération au développement organisée selon ces buts devrait être d'autre part complétée par un programme de recherche national ayant pour mission d'étudier les stratégies de développement pour les années 1990.

La "Société d'études Suisse - Tiers Monde" a été fondée en février 1984, afin de discuter des relations complexes existant entre la Suisse et le Tiers Monde. Le président de cette société d'études est E.A. Brugger. Ses membres, provenant des milieux de la science, de l'économie et des organismes de développement, se proposent de travailler ensemble dans des groupes d'études sur les questions prioritaires concernant ces relations : endettement, relations commerciales, évaluation de divers types de projets.

\section{Sources}

Rapport de gestion du Conseil fédéral, 1983.

Rapport annuel de la DDA, 1983.

Message concernant la continuation de la coopération technique et de l'aide financière en faveur des pays en développement, 19 mars 1984.

Bulletin officiel de l'Assemblée fédérale.

Plan financier 1984-1986 (DFF).

Actualités i3m (Service d'information Tiers Monde, Lausanne).

EVD-Pressemitteilungen, 20.7.83, 16.5.84.

EVD-Pressemitteilung, 16.1.84.

Pressecommuniqué "Für eine Welt ohne Hunger", 21.6.84.

Swissaid, Schweiz, Hilfe für Entwicklungsländer, Bern, 1984.

NZZ, 17.2., 14.4., 23.5.84.

Tages-Anzeiger, 23.5.84. 